\title{
A Survey on Energy Efficiency in Cellular Networks
}

\author{
Xiaochen Su${ }^{1}$, Enchang Sun ${ }^{1}$, Meng $\mathrm{Li}^{1}$, F. Richard $\mathrm{Yu}^{1,2}$, Yanhua Zhang ${ }^{1}$ \\ ${ }^{1}$ College of Electronic Information and Control Engineering, Beijing University of Technology, Beijing, China \\ ${ }^{2}$ Department of Systems and Computer Eng., Carleton University, Ottawa, ON, Canada \\ Email: hinata05269@sina.com, ecsun@bjut.edu.cn
}

Received July 2013

\begin{abstract}
With the continuous increase of user requirement, the Information and Communication Technology (ICT) has developed rapidly. As a result, both capacity and coverage performances have been improved, and at the same time, the communication networks have been more energy-intensive. In order to improve the energy efficiency of cellular networks, the green communication must be realized in the future networks. In this article, we present a short survey on energy-efficient technology of cellular networks. Moreover, we classify them into three categories based on their application scenarios: energy-efficient architectures, energy-efficient resource management and energy-efficient radio technologies. For the first scenario, the applications of relay, Coordinated Multiple Points (CoMP) and heterogeneous network are discussed in detail. For the second scenario, the switching off scheme will be introduced as an emphasis of this part. And for the third scenario, the Multiple-Input Multiple-Output (MIMO) and Orthogonal Frequency Division Multiplexing (OFDM) will be introduced as the representatives of the Energy-Efficient Radio Technologies. Finally, based on the technologies introduced above, a prospect forecast of the energy-efficient wireless cellular network is presented.
\end{abstract}

Keywords: Green Wireless Communication; Energy-Efficient; CoMP; Relay; Switching Off Scheme

\section{Introduction}

In the last several decades, due to the continuous increase of communication requirement, the Information and Communication Technology (ICT) developed rapidly focused on the higher capacity and larger coverage [1]. And correspondingly, the energy consumption is also growing at an amazing speed: the ICT is responsible for about $3 \%$ of energy consumption and $2 \%-4 \%$ of $\mathrm{CO}_{2}$ emission all over the world [2,3], and the energy consumption will increase at a rate of $15 \%$ - $20 \%$ per year and will double after 5 years [4]. Within the wireless cellular networks, the Base Station (BS) occupies a significant portion of energy about $60 \%-80 \%$, which means a breakthrough point to decrease the energy consumption [5].

In order to reduce the energy consumption, several organizations or projects, such as the Energy Aware Radio and Network Technologies (EARTH), have been founded, and many workshops have been organized at international conference [6,7]. Due to the endeavor and attempt for several years, various significant methods have been proposed.

In this paper, we present a survey on energy-efficient technology within the cellular network. The technologies can be classified into three categories according to their application scenarios. And for each aspect, the representative technologies are introduced and discussed further.
After that, a prospect forecast of the energy-efficient wireless cellular network is presented.

The rest of this paper is organized as follows. In Section 2, the energy-efficient architecture is introduced. The energy-efficient resource management is discussed in Section 3. In Section 4, some Radio Technologies are discussed. Within Section 5, the prospect forecast is presented. Finally, Section VI concludes this paper.

\section{Energy-Efficient Architectures}

A rational architecture is very important to reduce the energy consumption since it has a comprehensive influence on networks. In order to optimize the network architecture, a mass of methods based on some technologies, such as the heterogeneous network, CoMP and relay, have been proposed.

With deploying some special cells, such as microcells, picocells and femtocells, to assist the conventional macro cells, the heterogeneous network will increase coverage of the cellular networks as well as EE [8]. In [9], the authors analyze the energy consumption of heterogeneous network, and obtain a higher EE based on a balance point they found between coverage range and outage probability. In [10], the trade-off between SE and EE within the heterogeneous networks is studied, and in [11], the tradeoff between capacity and EE of heterogeneous network is 
studied deeply and the authors obtain a result that the small cell has a good potential to improve the EE and network capacity at the same time. In [12,13], the foremost trade-off between the deployment efficiency (DE) and EE within heterogeneous network is investigated. With their analysis of deployment density, the proper cell size and number of BSs have been presented as a reference for future design.

With the cooperation of multiple nodes, CoMP can increase the coverage and throughput of cellular networks, and the mobile users can be served with a relatively stable performance and quality even located at other cells [14]. In [15], the capacity and EE of an idealized CoMP system are analyzed by assuming the perfect backhauling and cooperative processing. In [16], the authors study the trade-off between EE and SE within the uplink of CoMP system and drive their power consumption models for both idealized and realistic scenario. And in [17], the authors investigate the realistic measurement method of EE for various downlink CoMP methods in LTE-A, and their results indicate that the coordinated beamforming and joint transmission could be the more suitable techniques to improve the EE. In [18,19], the ideas of switching off scheme and sleeping mode are combined with CoMP respectively to reduce the energy consumption further, and the switching off scheme and sleeping mode will be introduced primarily in the next section. In [20], the authors present a stochastic predictive control algorithm to optimize the BSs grouping for EE in CoMP. And in [21], a cooperative framework is proposed to dynamically decide whether to perform CoMP transmission or not. Except the researches above, in [22], the authors present a transmission mode selection scheme to save energy within the CoMP system using Semi-Smart Antenna. Within the transmission mode selection scheme, the conventional transmission mode, CoMP communication, transmitting with SSA, and the transmission with both CoMP and SSA are involved.

As same as CoMP, the relayed technology can also provides extra coverage and throughput to the conventional cellular networks and improve the communication quality within the cell edge. In [23], the energy-saving performance of CoMP and relay, affected by the traffic intensity and network density, are compared based on the typical parameters setting. The comparing result is varying with different intensity. In [24], the authors verify the
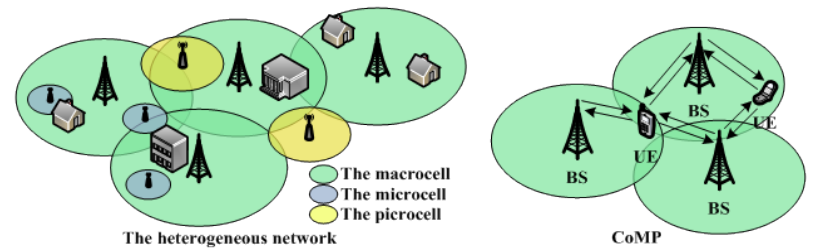

Figure 1. Model of heterogeneous network and CoMP. beneficial effect on EE by deploying the fixed Relay Stations (RS). The result within this paper shows that the energy minimization will not decrease the Signal to Interference Noise Ratio (SINR) of cellular relay network. In [25], the EE of cellular network with Realistic system characteristics and Amplify and Forward (AF) relay is investigated, and by using the convolution coding before transmission, the EE will be increased at high Signal to Noise Ratio (SNR) scenario. In [26], the scenario of Decode and Forward (DF) relay and Direct Transmission (DT) are also investigated. For a given outage probability, the network coding is beneficial for the situation with higher cell radius. In [27-32], the relay selection characterized on EE, such as taking the optimal locations of nodes to minimum transmitting power, and the instantaneous channel state obtained by channel estimation into account respectively in $[27,30]$, has been investigated to reduce the energy consumption further. Moreover, the research result within [32] shows that the dynamic time allocation and increasing the cooperative relays within the transmitting procedure properly are also beneficial to EE. In addition, based on the relay selection, the selection of transmission modes can improve the EE further. In [33], a self-adaptive energy-efficient transmission scheme is presented to choose the most suitable transmission mode based on the EE calculated before. Furthermore, the number of relays deployed in cells is also important to the EE. In [34], the authors investigate the trade-off between EE and deployment density deeply. The analysis shows that a proper deployment density of relays will satisfy the requirement both of EE and DE. Too many relays will lower the EE and increase the deploying cost as well.

In the next generation cellular networks, CoMP will play a crucial role in improving the communication quality and EE at the same time. Therefore, the joint consideration of CoMP and other technologies needs to be put into more attention. And the optimized deployment of small cells and relays for energy efficiency is still an open issue needed to be studied further.

\section{Energy-Efficient Resource Management}

Although the improved architecture can bring some benefits, the corresponding resource management is also indispensable to realize the green communication. For this reason, several technologies have been taken into account the energy efficient resource management, such as the switching off scheme, cell zooming, the using of renewable energy and so on.

In traditional cellular networks, the operators deployed many BSs to cover the communication blind district and improve the communication quality. Although it really works sometimes, plenty energy has been wasted due to 
the low utilization of BSs. For this reason, many switching off schemes based on the variation of traffic load have been proposed in [35-42]. Except the common criterion presented above, the distance between the User Equipments (UEs) and the BSs mentioned in [35], the additional load increments transferred to the adjacent BSs considered in [37], the maximized coverage provided by active BSs and the coverage overlap of BSs adopted in $[39,42]$ respectively are also the important criterions that should be taken into account. Besides the operation in BS, the switching off scheme can also be applied in relay networks. Although the transmitting power of RSs is much lower than BSs, RSs with lower utilization will also cause the energy wastage. In [43], the relays are dynamically switched off according to the variation of traffic loads. And in [44], the throughput and energy consumption are treated with the criterions simultaneously to decide which RSs should be switched off to improve EE of the network.

As a power control technology, the cell zooming is used to satisfy the demand of traffic load in general, but can also increase EE of cellular networks. And in most instances, cell zooming is adopted as an assistant technology to improve the EE with other technologies. In $[45,46]$, the authors put the low utilization BS into sleeping mode (operating with a low energy level) and cover the uncovered area by using cell zooming. And in $[47,48]$, the cell zooming is combined with the switching off scheme to minimize the energy consumption.

Except the technologies introduced above, the usage of renewable energy can also reduce the electric energy consumption more directly. In [49], a handover method is presented to guide the users to access the BSs powered by green energy. In [50], the authors propose a scheme to enable more users to be served by the green energy through cell zooming. In [51], the authors investigate a system model with BSs powered by the combination of electronic and renewable energy. And In [52], the authors combine the BS switching off scheme with hybrid power to further increase the EE.

Although the renewable energy can reduce the electric energy consumption directly, the study of this aspect is beyond our research. For this reason, to our best knowledge, the switching off scheme is considered as the most effective method to manage the resource within future
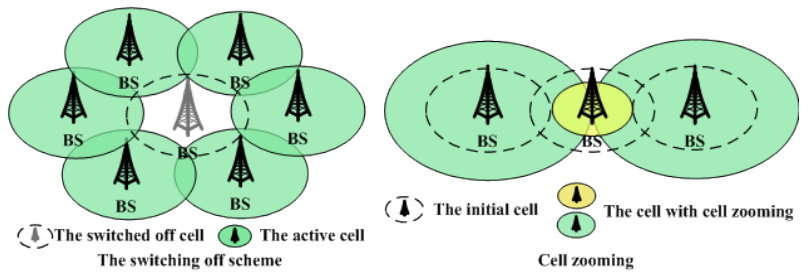

Figure 2. The switching off scheme and cell zooming. networks. Moreover, the switching off scheme can also be combined with CoMP, cell zooming and/or other technologies to maintain a better coverage and improve the $\mathrm{EE}$ at the same time. Therefore, the joint switching off scheme needs further investigation.

\section{Energy-Efficient Radio Technologies}

The green radio technology can effectively reduce the energy consumption within the transmitting procedure, and then improve the EE of total network. In this section, MIMO and OFDM will be introduced as representatives.

Due to the noteworthy improvement on average data rate and SE, MIMO has drawn many attentions within cellular networks. And pushed by the trend of green communication, the EE of MIMO is also becoming a research hotspot. In [53], the trade-off between energy and bandwidth efficiency is studied within a MIMO multihop wireless networks. And the analysis results show that the number of transmit antennas, receive antennas and communication hops are responsible to improve EE. In [54], the EE of different MIMO transmission schemes, such as the Open Loop Spatial Multiplexing (OLSM) and Close Loop Spatial Multiplexing (CLSM), have been investigated and compared with the traditional SISO mode. And in [55], the authors prove that the feedback information is very important to improve the throughput and EE especially in the CLSM which utilizes all available feedback information. In addition, for the case of the UE has only one antenna in general, the energy-efficient MUMIMO has been investigated in [56,57]. In [56], the authors devote themselves to balance the cell-edge EE and the average EE within cell. And in [57], the emphasis is put on the balance between EE and capacity by improving the power allocation.

As same as MIMO, OFDM also has a good potentiality to reduce the energy consumption. In [58,59], some important trade-offs are studied in the OFDMA downlink. In [58], the authors prove that the relationship between $\mathrm{EE}$ and $\mathrm{SE}$ is quasi-concave and they find the upper bound and lower bound on the EE-SE curve for general scenarios. In [59], the authors propose an algorithm to increase the EE and reduce the computation overhead properly at the same time. Except these fundamental researches,

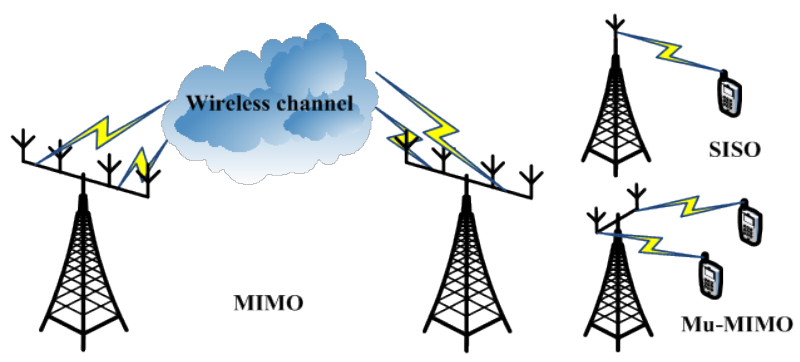

Figure 3. The scenarios of SISO, MIMO and Mu-MIMO. 
OFDM is often combined with the power and resource allocation to improve EE further. In [60], the authors improve EE by optimizing the number of sub-carriers. And in [61], the overhead of CSI feedback is reduced to achieve higher EE.

Although the research aimed at improving EE of MIMO and OFDM systems have been continued for years, there are many aspects, such as the MU-MIMO and multi-cell OFDM, need to be investigated more deeply.

\section{Prospect Forecast}

Based on the introduction and discussion of the energyEfficient Technologies above, to our best knowledge, an optimized energy-efficient wireless cellular network can be conceived. According to the fact that the BSs occupy a significant portion of energy consumption, the heterogeneous network can be used to reduce the energy consumption while providing a better communication performance. Besides, the combination of heterogeneous network and switching off scheme will further increase the EE. In order to avoid the frequent switching, the low utilization BSs or RSs can be turned into a sleeping mode first. If the low utilization lasts for a long time, the sleeping nodes will be switched off completely. And as the important supplement, the cell zooming and CoMP can be adopted to cover the communication blind district. For a more optimized consideration, the OFDM and MIMO should be introduced into the signal transmission due to their better performance of balancing the EE and other criterions such as SE and capacity.

\section{Conclusion}

In this article, we present a short survey on energy-efficient technologies of cellular networks. Many hotspot technologies such as the CoMP, MIMO and switching off scheme are introduced as the representatives, which are divided into three categories according to their application scenarios. For each part, the current research situations of technologies are introduced and discussed in detail. And to our best knowledge, a prospect forecast of energy efficient network is presented.

\section{Acknowledgements}

This work was jointly supported by the National Natural Science Foundation of China under Grant 61101113, 61072088 and 61201198, the Beijing Natural Science Foundation of China under Grant 4132019, 4132015 and 4132007, and the Doctorate Subject Foundation of the Ministry of Education under Grant 20111103120017.

\section{REFERENCES}

[1] D. Q. Feng, C. Z. Jiang, G. B. Lim, L. J. Cimini, Jr., G.
Feng and G. Y. Li, "A Survey of Energy-Efficient Wireless Communications,” Communications Surveys \& Tutorials, IEEE, Vol. 15, No. 1, 2013, pp. 167-178. http://dx.doi.org/10.1109/SURV.2012.020212.00049

[2] M. Pejanovic-Djurisic, E. Stovrag and M. Ilic-Delibasic, "Fundamental Optimization Criteria For Green Wireless Communications," MIPRO, 2012 Proceedings of the 35th International Convention, Opatija, 21-25 May 2012, pp. 733-736.

[3] A. Amokrane, R. Langar, R. Boutaba and G. Pujolle, “A Green Framework for Energy Efficient Management in TDMA-based Wireless Mesh Networks," Network and Service Management (CNSM), 2012 8th International Conference and 2012 Workshop on Systems Virtualiztion Management (SVM), Las Vegas, NV, 22-26 October 2012, pp. 322-328.

[4] K. Son, H. Kim, Yung Yi and B. Krishnamachari, "Base Station Operation and User Association Mechanisms for Energy-Delay Tradeoffs in Green Cellular Networks," IEEE Journal on Selected Areas in Communications, Vol. 29, No. 8, 2011, pp. 1525-1536.

http://dx.doi.org/10.1109/JSAC.2011.110903

[5] H. Bogucka and A. Conti, "Degrees of Freedom for Energy Savings in Practical Adaptive Wireless Systems," IEEE Communications Magazine, Vol. 49, No. 6, 2011, pp. 38-45. http://dx.doi.org/10.1109/MCOM.2011.5783983

[6] G. Y. Li, Z. k. Xu, C. Xiong, C. Y. Yang, S. Q. Zhang, Y. Chen and S. G. Xu. "Energy-Efficient Wireless Communications: Tutorial, Survey and Open Issues," IEEE Wireless Communication, Vol. 18, No. 6, 2011, pp. 28-35. http://dx.doi.org/10.1109/MWC.2011.6108331

[7] S. Tombaz, A. Vastberg and J. Zander, "Energy and Cost Efficient Ultra High Capacity Wireless Access," IEEE Wireless Communication Magazine, Vol. 18, No. 5, 2011, pp. 18-24.

http://dx.doi.org/10.1109/MWC.2011.6056688

[8] M. Nasimi, F. Hashim and C. K. Ng, "Characterizing Energy Efficiency for Heterogeneous Cellular Networks,” Research and Development (SCOReD), 2012 IEEE Student Conference on, Pulau Pinang, 5-6 December 2012, pp. 198-202.

[9] A. Singh and R. Mahapatra, "Energy Efficient Analysis of Heterogeneous Wireless Network," Advance Computing Conference (IACC), 2013 IEEE 3rd International, Ghaziabad, 22-23 February 2013, pp. 531-535.

[10] R. Amin, J. Martin, A. Eltawil and A. Hussien, "Spectral Efficiency and Energy Consumption Tradeoffs for Reconfigurable Devices in Heterogeneous Wireless Systems," Wireless Communications and Networking Conference (WCNC), 2012 IEEE, Shanghai, 1-4 April 2012, pp. 1698-1703.

[11] L. Saker, S. E. Elayoubi, T. Chahed and A. Gati, "Energy Efficiency and Capacity of Heterogeneous Network Deployment in LTE-Advanced," European Wireless, 2012. EW. 18th European Wireless Conference, Poznan, Poland 18-20 April 2012, pp. 1-7.

[12] G. N. He, S. Q. Zhang, Y. Chen and S. G. Xu, "Energy Efficiency and Deployment Efficiency Tradeoff for Heterogeneous Wireless Networks,” Global Communications 
Conference (GLOBECOM), 2012 IEEE, Anaheim, CA, 3-7 December 2012, pp. 3189-3194. http://dx.doi.org/10.1109/GLOCOM.2012.6503605

[13] B. Lee and S.-L. Kim, "Characterizing Energy and Deployment Efficiency Relations in Cellular Systems,” Signal Processing and Communication Systems (ICSPCS), 2012 6th International Conference on, Gold Coast, QLD, 12-14 December 2012, pp. 1-5.

[14] J. Thompson and C. Khirallah, "Overview of Green Radio Research Outcomes," Communications in China Workshops (ICCC), 2012 1st IEEE International Conference on, Bejing, 15-17 August 2012, pp. 69-73.

[15] F. Heliot, M. A. Imran and R. Tafazolli, "Energy Efficiency Analysis of Idealized Coordinated Multi-Point Communication System," Vehicular Technology Conference (VTC Spring), 2011 IEEE 73rd, Yokohama, 15-18 May 2011, pp.1-5.

[16] O. Onireti, F. Heliot and M. A. Imran, “On the Energy Efficiency-Spectral Efficiency Trade-Off in the Uplink of CoMP System," Wireless Communications, IEEE Transactions on, Vol. 11, No. 2, 2012, pp. 556-561. http://dx.doi.org/10.1109/TWC.2011.120911.111494

[17] K. M. S. Huq, S. Mumtaz, J. Rodriguez and R. L. Aguiar, "Comparison of Energy-Efficiency in Bits per Joule on different downlink CoMP Techniques," Communications (ICC), 2012 IEEE International Conference on, Ottawa, ON, 10-15 June 2012, pp. 5716-5720.

[18] G. Cili, H. Yanikomeroglu and F. R. Yu, "Cell Switch Off Technique Combined with Coordinated Multi-Point (CoMP) Transmission for Energy Efficiency in BeyondLTE Cellular Networks," Communications (ICC), 2012 IEEE International Conference on, Ottawa, ON, 10-15 June 2012, pp. 5931-5935.

[19] K. M. S. Huq, S. Mumtaz, M. Alam, A. Radwan and J. Rodriguez, "Energy Efficient CoMP Transmission in LTE-Advanced,” Globecom Workshops (GC Wkshps), 2012 IEEE, Anaheim, CA, 3-7 December 2012, pp. 401404.

[20] S. H. Wen and F. R. Yu, "Predictive Control for Energy Efficiency in Wireless Cellular Networks," Vehicular Technology Conference (VTC Spring), 2012 IEEE 75th, Yokohama, 6-9 May 2012, pp. 1-5.

[21] F. Pantisano, M. Bennis, W. Saad, R. Verdone and M. Latva-aho, "On the Dynamic Formation of Cooperative Multipoint Transmissions in Small Cell Networks," Globecom Workshops (GC Wkshps), 2012 IEEE, Anaheim, CA, 3-7 December 2012, pp. 1139-1144.

[22] Y. T. Zhu, Z. M. Zeng, T. K. Zhang and L. Xiao, “Transmission Mode Selection for Energy Saving in The CoMP System with Semi-Smart Antenna," Communications and Information Technologies (ISCIT), 2012 International Symposium on, Gold Coast, QLD, 2-5 October 2012, pp. 855-860.

[23] D. X. Cao, S. Zhou, C. Zhang and Z. S. Niu, "Energy Saving Performance Comparison of Coordinated MultiPoint Transmission and Wireless Relaying," Global Telecommunications Conference (GLOBECOM 2010), 2010 IEEE, Miami, FL, 6-10 December 2010, pp. 1-5.

[24] G. Chandwani, S. N. Datta and S. Chakrabarti, "Relay
Assisted Cellular System for Energy Minimization,” India Conference (INDICON), 2010 Annual IEEE, Kolkata, 17-19 December 2010, pp. 1-4. http://dx.doi.org/10.1109/INDCON.2010.5712641

[25] M. Al-Kali, Y. Li, D. Samb, C. Liu and D. S. Wang, "Performance Analysis for Energy Efficiency in Wireless Cooperative Relay Networks," Communication Technology (ICCT), 2012 IEEE 14th International Conference on, Chengdu, 9-11 November 2012, pp. 423-427.

[26] T. ur R. Ahsin and S. B. Slimane, “Area Energy Consumption in Cooperative Decode and Forward (DF) Relaying Scenarios,” Wireless Conference 2011, Sustainable Wireless Technologies (European Wireless), 11th European, Vienna, Austria, 27-29 April 2011, pp. 1-8.

[27] Z. Z. Tang, H. Y. Wang and Q. Hu, “An Energy-Efficient Relay Selection Strategy Based on Optimal Relay Location for AF Cooperative Transmission," World of Wireless, Mobile and Multimedia Networks (WoWMoM), 2012 IEEE International Symposium on, San Francisco, CA, 25-28 June 2012, pp.1-4.

[28] M. Zhou, Q. M. Cui, R. Jantti and X. F. Tao, "EnergyEfficient Relay Selection and Power Allocation for TwoWay Relay Channel with Analog Network Coding," Communications Letters, IEEE, Vol. 16, No. 6, 2012, pp. 816-819.

http://dx.doi.org/10.1109/LCOMM.2012.040912.112435

[29] J. H. Lee, Y. M. Lim, K. Kim, S. G. Choi and J. K. Choi, "Energy Efficient Cooperative Multicast Scheme Based on Selective Relay," Communications Letters, IEEE, Vol. 16, No. 3, 2012, pp. 386-388. http://dx.doi.org/10.1109/LCOMM.2012.011312.112375

[30] Y. F. Wei, C. W. Wang, M. Song, Y. Ma and X. J. Wang, "Energy Saving Dynamic Relaying Scheme in Wireless Cooperative Networks Using Markov Decision Process," Vehicular Technology Conference (VTC Spring), 2012 IEEE 75th, Yokohama, 6-9 May 2012, pp. 1-5.

[31] D. Chen, H. Ji and X. Li, “An Energy-Efficient Distributed Relay Selection and Power Allocation Optimization Scheme over Wireless Cooperative Networks," Communications (ICC), 2011 IEEE International Conference on, Kyoto, 5-9 June 2011, pp. 1-5.

[32] O. Amin and L. Lampe, "Opportunistic Energy Efficient Cooperative Communication,” Wireless Communications Letters, IEEE, Vol. 1, No. 5, 2012, pp. 816-819. http://dx.doi.org/10.1109/WCL.2012.061212.120206

[33] T. Kang, X. K. Sun, Y. T. Zhu and T. K. Zhang, “A Self-adaptive Energy Efficient Transmission Mechanism in Relay Based Cellular Network," Communications and Information Technologies (ISCIT), 2012 International Symposium on, Gold Coast, QLD, 2-5 October 2012, pp. 861-866.

[34] C. Khirallah, J. S. Thompson and H. Rashvand, "Energy and Cost Impacts of Relay and Femtocell Deployments in Long-Term-Evolution Advanced," Communications, IET, Vol. 5, No. 18, 2011, pp. 2617-2628. http://dx.doi.org/10.1049/iet-com.2011.0111

[35] A. Bousia, E. Kartsakli, L. Alonso and C. Verikoukis, "Dynamic Energy Efficient Distance-Aware Station Switch On-Off Scheme for LTE-Advanced," Global 
Communications Conference (GLOBECOM), 2012 IEEE, Anaheim, CA, 3-7 December 2012, pp. 1532-1537.

[36] X. Chen, Z. Feng and D. Yang, “An Energy-Efficient Macro-Micro Hierarchical Structure with Resource Allocation in OFDMA Cellular Systems," Communications and Networking in China (CHINACOM), 2012 7th International ICST Conference on, Kun Ming , 8-10 August 2012, pp. 519-523.

[37] O. H. Eunsung, S. Kyuho and B. Krishnamachari, "Dynamic Base Station Switching-On Off Strategies for Green Cellular Networks," Wireless Communications, IEEE Transactions on, Vol. 12, No. 5, 2013, pp. 21262136. doi:10.1109/TWC.2013.032013.120494

[38] W. Guo and T. O’Farrell, “Dynamic Cell Expansion Traffic Aware Low Energy Cellular Network," Vehicular Technology Conference (VTC Fall), 2012 IEEE, Quebec City, 3-6 September 2012, pp. 1-5.

[39] H.-S. Jung, H.-T. Roh and J.-W. Lee, "Energy and Traffic Aware Dynamic Topology Management for Wireless Cellular Networks,” Communication Systems (ICCS), 2012 IEEE International Conference on, Singapore, 21-23 November 2012, pp. 205-209.

[40] Y. Wu and Z. Niu, "Energy Efficient Base Station Deployment in Green Cellular Networks with Traffic Variations," Communications in China (ICCC), 2012 1st IEEE International Conference on, Beijing, 15-17 August 2012, pp. 399-404.

[41] A. Bousia, E. Kartsakli, L. Alonso and C. Verikoukis, "Energy Efficient Base Station Maximization Switch Off Scheme for LTE-Advanced,” Computer Aided Modeling and Design of Communication Links and Networks (CAMAD), 2012 IEEE 17th International Workshop on, Barcelona, 17-19 September 2012, pp. 256-260.

[42] L. Chiaraviglio, D. Ciullo, G. Koutitas, M. Meo and L. Tassiulas, "Energy-Efficient Planning and Management of Cellular Networks," Wireless On-demand Network Systems and Services (WONS), 2012 9th Annual Conference on, Courmayeur, 9-11 January 2012, pp. 159-166.

[43] D. Quintas and V. Friderikos, "On Dynamic Policies to Switch Off Relay Nodes,” Communications (ICC), 2012 IEEE International Conference on, Ottawa, 10-15 June 2012, pp. 2945-2950.

[44] S.-Y. Lee and D.-N. Yang, “Joint Selection of On/off Relay Mode and Adaptive Modulation Mode for Green Cooperative Multicast Networks," Vehicular Technology Conference (VTC Fall), 2012 IEEE, Quebec City, 3-6 September 2012, pp. 1-5.

[45] G. Micallef, P. Mogensen and H.-O. Scheck, "Cell Size Breathing and Possibilities to Introduce Cell Sleep Mode," Wireless Conference (EW), 2010 European, Lucca, 12-15 April 2010, pp. 111-115. doi:10.1109/EW.2010.5483401

[46] L. A. Suarez, L. Nuaymi and J. Bonnin, "Energy Performance of a Distributed BS Based Green Cell Breathing Algorithm," Wireless Communication Systems (ISWCS), 2012 International Symposium on, Paris, 28-31 August 2012, pp. 341-345.

[47] X. Weng, D. Cao and Z. Niu, "Energy-Efficient Cellular
Network Planning under Insufficient Cell Zooming," Vehicular Technology Conference (VTC Spring), 2011 IEEE 73rd, Yokohama, 15-18 May 2011, pp. 1-5.

[48] P. Yu, Q. Yang, F. Fu and K. S. Kwak, "Inter-Cell Cooperation Aided Dynamic Base Station Switching for Energy Efficient Cellular Networks," Communications (APCC), 2012 18th Asia-Pacific Conference on, Jeju Island, 15-17 October 2012, pp.159-163.

[49] J. Zhou, M. Li, L. Liu, X. She and L. Chen, "Energy Source Aware Target Cell Selection and Coverage Optimization for Power Saving in Cellular Networks,” Green Computing and Communications (GreenCom), 2010 IEEE/ACM Int'l Conference on \& Int'l Conference on Cyber, Physical and Social Computing (CPSCom), Hangzhou, 18-20 December 2010, pp. 1-8.

[50] H. Tao and N. Ansari, "ICE Intelligent Cell BrEathing to Optimize the Utilization of Green Energy," Communications Letters, IEEE, Vol. 16, No. 6, 2012, pp. 866-869. doi:10.1109/LCOMM.2012.042312.120486

[51] D. Niyato, X. Lu and P. Wang, “Adaptive Power Management for Wireless Base Stations in a Smart Grid Environment," Wireless Communications, IEEE, Vol. 19, No. 6, 2012, pp. 44-51. doi:10.1109/MWC.2012.6393517

[52] H. Ghazzai, E. Yaacoub, M. Alouini and A. Abu-Dayya, "Optimized Green Operation of LTE Networks in the Presence of Multiple Electricity Providers,” Globecom Workshops (GC Wkshps), 2012 IEEE, Anaheim, 3-7 December 2012, pp. 664-669.

[53] C.-L. Chen, W. E. Stark and S.-G. Chen, "Energy-Bandwidth Efficiency Tradeoff in MIMO Multi-Hop Wireless Networks," Selected Areas in Communications, IEEE Journal on, Vol. 29, No. 8, 2011, pp. 1537-1546. doi:10.1109/JSAC.2011.110904

[54] O. Adigun, and C. Politis, "Energy Efficiency Analysis for MIMO Transmission Schemes in LTE,” Computer Aided Modeling and Design of Communication Links and Networks (CAMAD), 2011 IEEE 16th International Workshop on, Kyoto, 10-11 June 2011, pp. 77-81.

[55] O. Adigun, and C. Politis, "Feedback Information and Energy Efficiency of MIMO Transmission Modes in LTE,” Sarnoff Symposium (SARNOFF), 2012 35th IEEE, Newark, 21-22 May 2012, pp. 1-5.

[56] L. Liu, G. Miao and J. Zhang, "Energy-Efficient Scheduling for Downlink Multi-User MIMO,” Communications (ICC), 2012 IEEE International Conference on, Ottawa, 10-15 June 2012, pp. 4390-4394.

[57] G. Miao and J. Zhang, "On Optimal Energy-Efficient Multi-User MIMO,” Global Telecommunications Coference (GLOBECOM 2011), 2011 IEEE, Houston, 5-9 December 2011, pp. 1-6.

[58] C. Xiong, G. Y. Li, S. Zhang, Y. Chen and S. Xu, "Energy- and Spectral-Efficiency Tradeoff in Downlink OFDMA Networks," Wireless Communications, IEEE Transactions on, Vol. 10, No. 11, 2011, pp. 3874-3886. doi:10.1109/TWC.2011.091411.110249

[59] F.-S. Chu and K.-C. Chen, "Energy Efficient OFDMA: Trade-Off between Computation and Transmission Energy,” Personal Indoor and Mobile Radio Communica- 
tions (PIMRC), 2011 IEEE 22nd International Symposium on, Toronto, 11-14 September 2011, pp. 96-101.

[60] C. Sun, Y. Cen and C. Yang, "Energy Efficient OFDM Relay Systems," Communications, IEEE Transactions on, Vol. 61, No. 5, 2013, pp. 1797-1809. doi:10.1109/TCOMM.2013.021913.120555
[61] C. Xiong, G. Y. Li, S. Zhang and S. Xu, "CSI Feedback Reduction for Energy-Efficient Downlink OFDMA," Wireless Communications and Networking Conference (WCNC), 2012 IEEE, Shanghai, 1-4 April 2012, pp. 1135-1139. 